\title{
Local Drug Delivery by Fibrin Glue for Glioma Treatment: Enhancing Drug Efficacy by Photochemical Internalization (PCl)
}

\author{
Lina Nguyen ${ }^{1 *}$, Diane Shin ${ }^{1}$, Mai T Le ${ }^{1}$, Eric O Potma ${ }^{1}$, Nehal Idris $^{1}$, Jimmy Nguyen Le $^{1}$, Julie Johnson ${ }^{1}$, \\ Qian Peng ${ }^{2}$, Kristian Berg ${ }^{3}$ and Henry Hirschberg ${ }^{1}$
}

${ }^{1}$ Beckman Laser Institute and Medical Clinic, University of California, United States

${ }^{2}$ Department of Pathology, The Norwegian Radium Hospital, Oslo University Hospital, Norway

${ }^{3}$ Department of Radiation Biology, The Norwegian Radium Hospital, Oslo University Hospital, Norway

\begin{abstract}
Background: Localized methods of drug delivery, such as controlled drug release from fibrin glue (FG), has the potential of improving the efficacy of chemotherapy for brain tumors by bypassing the blood brain barrier. Photochemical internalization ( $\mathrm{PCl}$ ) of bleomycin (BLM) and doxorubicin (DOX) has been shown to enhance the cytosolic delivery of drugs in localized site and temporal specific manner. This study examined the ability of $\mathrm{PCl}$ to enhance the growth inhibiting effects of BLM or DOX released from FG on multi-cell glioma spheroids in vitro.

Materials and Methods: FG layers consisted of a 1:1 ratio of fibrinogen and drug or photosensitizer loaded thrombin. Supernatants covering the FG layers were harvested from the gelled FG containing wells after 2, 24, 48 and 72 hours. F98 glioma cells in suspension were incubated with the photosensitizer aluminum phthalocyanine disulfonate (AlPcS 2 , $1 \mu \mathrm{g} /$ $\mathrm{mL}$ ) for 2 hours, washed and formed as 3D spheroids. The spheroids were incubated with BLM or DOX released from FG. One hour later, the spheroids were treated with light $(\mathrm{PCl}), \lambda=670 \mathrm{~nm}$, from a diode laser at an irradiance of $2.0 \mathrm{~mW} /$ $\mathrm{cm}^{2}$. Spheroid growth was monitored for an additional 14 days.
\end{abstract}

Results: At the BLM or DOX concentrations used in these experiments to load the FG, spheroid growth was not significantly influenced by the BLM or DOX that was released from the FG gels. In contrast, spheroid growth was significantly inhibited or completely suppressed by PCl of released drug from either FG-BLM or FG-DOX.

Conclusion: The results of the present study show that drug was released in a non-degraded form for an extended time period. The growth inhibition caused by either FG released BLM or DOX was significantly enhanced by IIPCS $_{2 a}$ mediated $\mathrm{PCl}$.

\section{Keywords}

Photochemical Internalization, PCl, Fibrin Glue, Bleomycin, Doxorubicin

\section{Introduction}

The Central Brain Tumor Registry shows that gliomas account for $34 \%$ of all primary brain tumors, and $82 \%$ of all malignant primary brain tumor cases. Despite continued efforts, glioblastoma multiforme (GBM) remains an incurable form of primary brain cancer. Even in cases of gross tumor resection (as determined from post-operative MRI), the tumor invariably recurs. In approximately $80 \%$ of all cases, recurrent tumor growth occurs within a $2-3 \mathrm{~cm}$ margin of the surgical resection cavity [1,2]. This is likely due to the migratory behavior of glioma cells - a trait attributed to their developmental character within the CNS. Both individual cells and micro-colonies of tumor cells have been shown to infiltrate in or beyond a region of brain-adjacent-to-tumor (BAT) - a zone that may extend several $\mathrm{cm}$ from the resection margin. Eradication of these infiltrating glioma cells poses a significant clinical challenge due to the fact that migratory tumor cells in

*Corresponding author: Dr. Lina Nguyen, Beckman Laser Institute and Medical Clinic, University of California, United States

Accepted: July 21, 2020

Published online: July 23, 2020

Citation: Nguyen L, Shin D, Le MT, et al. (2020) Local Drug Delivery by Fibrin Glue for Glioma Treatment: Enhancing Drug Efficacy by Photochemical Internalization (PCI). Insights Neurooncol 3(1):31-42 
Citation: Nguyen L, Shin D, Le MT, et al. (2020) Local Drug Delivery by Fibrin Glue for Glioma Treatment: Enhancing Drug Efficacy by Photochemical Internalization (PCI). Insights Neurooncol 3(1):31-42

and beyond the BAT are protected to varying degrees by the blood brain barrier (BBB). This formidable protective barrier unfortunately also severely limits the efficacy of systemically delivered chemotherapeutic and or nano-agents that cannot pass through the BBB.

One method of bypassing the BBB is the local application of chemotherapeutic drugs directly into the resection cavity during the surgical procedure. Localized drug delivery permits increasing the drug dose able to reach the remaining infiltrative tumor cells in the brain parenchyma while avoiding systemic side effects. For this type of local drug application to function adequately the drug should be delivered in a sustained release form. Several types of slow release vehicles have been evaluated for GBM treatment such as clinically approved BCNU biodegradable wafers [3] as well as various forms of hydrogels in experimental animals [4]. One form of hydrogel, fibrin glue (FG), has several characteristics that make it well suited for this form of drug delivery. In particular, it has been used clinically in surgery both as a sealant and to obtain hemostasis for decades, so its lack of toxicity has been clearly proven. FG loaded with drugs therefore has been re- searched as a localized controlled release vehicle [5-7].

FG consists of two components, a fibrinogen solution and a thrombin solution rich in calcium. When the two components are mixed, the thrombin enzymatically cleaves fibrinogen to form fibrin and factor XIII to factor XIIIa, which then crosslinks fibrin to form a gel. In the simplest formulation of fibrin glue as a drug delivery gel, exogenous drugs are added to one of the components before addition of the other, allowing the drug or factor to be distributed throughout the solution before cross linking. Since the amount of drug that can be loaded into FG has some limitations, methods to increase the efficacy of released anticancer agents are therefore of interest.

Photochemical internalization ( $\mathrm{PCl})$, a specialized form of photodynamic therapy (PDT) has been shown to improve the cytosolic delivery of drugs in a site-specific manner [8-10]. The basic principle for $\mathrm{PCl}$ is shown in Figure $1 \mathrm{a}$ and Figure $1 \mathrm{~b}$. Macromolecules that are internalized into cells via endocytosis end up inside and trapped in the intracellular endosomes and lysosomes (Figure 1a). The concept of $\mathrm{PCl}$ is based on

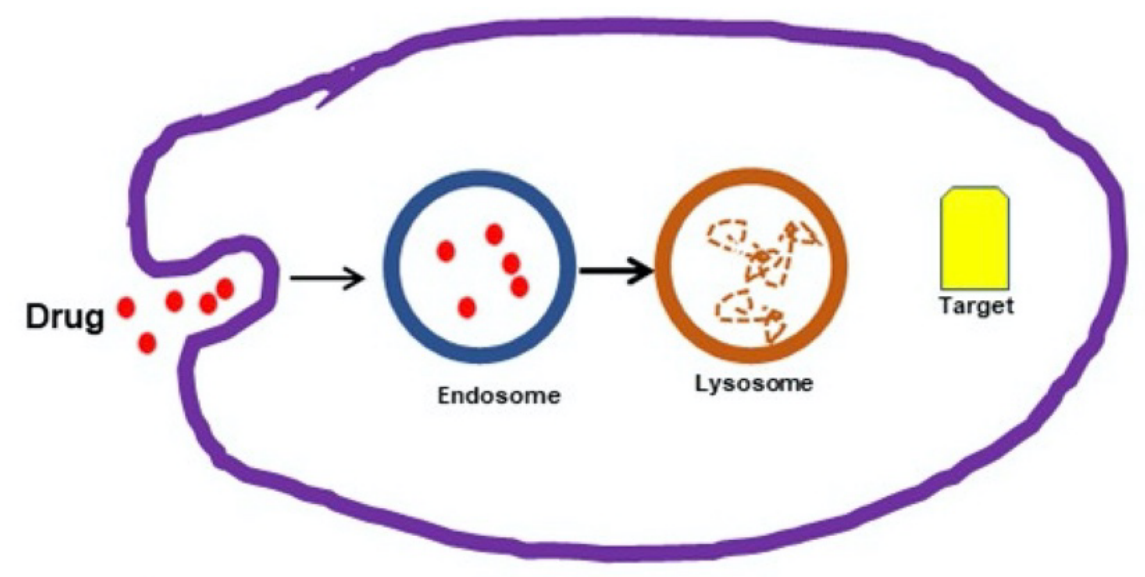

a

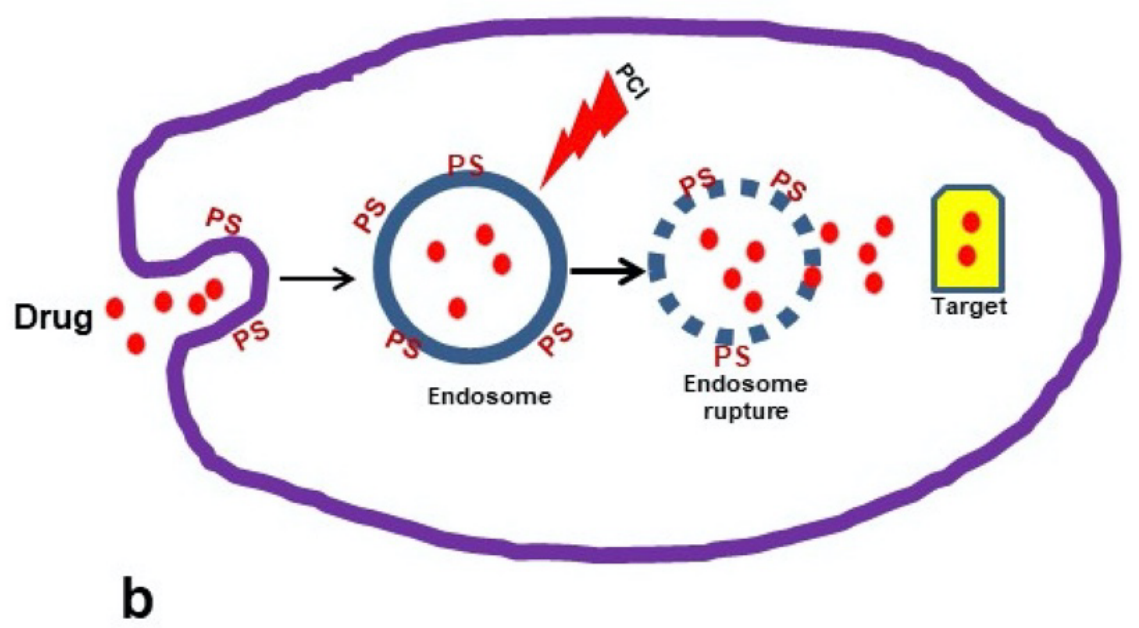

Figure 1: Schematic illustration of $\mathrm{PCl}$. a) No $\mathrm{PCl}$ : Drugs taken up through endocytosis are transported to endosomes which after fusion with lysosomes, are degraded before interacting with their targets; b) PCI: Photosensitizers are co-administered with drugs and accumulate in endosomes and lysosomes. Light exposure causes rupture of the endo/lysosomal membrane and releases the drugs into the cytosol where the drugs can exert their full biological effects. 
Citation: Nguyen L, Shin D, Le MT, et al. (2020) Local Drug Delivery by Fibrin Glue for Glioma Treatment: Enhancing Drug Efficacy by Photochemical Internalization (PCI). Insights Neurooncol 3(1):31-42

using specialized photosensitizers, which localize in the cell membrane, are carried into the cell during the endocytotic event and remain in the endosome membrane, with the macromolecule trapped inside the endosome lumen. Upon light exposure the photosensitizer interacts with ambient oxygen to produce singlet oxygen. Since singlet oxygen has a very short range of action $(<20 \mathrm{~nm}$ ), only the area of the vesicular membrane where the photosensitizer is localized will be damaged by singlet oxygen-mediated reactions with amino acids, unsaturated fatty acids and cholesterol in the membrane bilayer. The released agent can therefore exert its full biological activity, in contrast to being degraded by lysosomal hydrolases (Figure 1b).

$\mathrm{PCl}$ has been demonstrated to significantly enhance the efficacy of both bleomycin (BLM) and doxorubicin (DOX) [1115]. In particular, $\mathrm{PCl}$ of BLM has been demonstrated to be effective as an adjunct to inadequate surgery and could significantly delay tumor re-growth in a mouse tumor model, compared to PDT or BLM treatment alone [16]. The combination of local intra-cavity FG slow-release drug delivery combined with $\mathrm{PCl}$ therefore has the potential of bypassing the BBB allowing increased chemotherapeutic efficacy.

The aim of the present in vitro research is designed to evaluate the ability of $\mathrm{PCl}$ to enhance the effectiveness of FG released drug on multi-cell 3-dimensional tumor spheroids formed from glioma tumor cells [17]. As opposed to cell monolayers, tumor spheroids more closely mimic in vivo tumors in their micro-environment in terms of gene expression and the biological behavior of the cells in tumors. Their 3-dimensional structure creates oxygen, nutrient, and $\mathrm{pH}$ gradients that result in quiescent cells which are more resistant to chemotherapy, ionizing radiation, and PDT/PCI. Spheroid cultures are therefore considered to be a realistic bridge between monolayer in vitro culturing and tumor work in vivo.

\section{Materials and Methods}

\section{Cells}

The rat glioma line (F98) was obtained from the American Type Culture Collection (Manassas, VA, USA) and were maintained in Dulbecco's Modified Eagle Medium (DMEM) with high glucose (Life Technologies Corp., Carlsbad, CA, USA) supplemented with $2 \%$ fetal bovine serum (FBS), 25 mM HEPES buffer (pH 7.4), penicillin (100 $\left.\mathrm{U} \mathrm{mL}^{-1}\right)$ and streptomycin (100 $\left.\mu \mathrm{g} / \mathrm{mL}^{-1}\right)$ at $37^{\circ} \mathrm{C}$ and $5 \% \mathrm{CO}_{2}$.

\section{Chemicals}

The photosensitizer, aluminum phthalocyanine disulfonate $\left(\right.$ AlPcS $_{2 a}$ ) was obtained from Frontier Scientific, Inc. (Logan, UT, USA). The chemotherapeutic drugs Doxorubicin Hydrochloride (DOX) and Bleomycin (BLM) were obtained from Sigma Aldrich (St. Louis, MO, USA).

\section{Fibrin glue and drug harvest}

The fibrin glue was obtained from EMD Millipore Calbiochem (Temecula, CA, USA) and was composed of a 1:1 ratio of fibrinogen and thrombin, with drug or photosensitizer added to the thrombin. The $0.2 \mathrm{~mL}$ of the drug loaded thrombin was combined with $0.2 \mathrm{~mL}$ of fibrinogen in the wells of a 24 well microplate. The glue was allowed to gel for $20 \mathrm{~min}$ at $37^{\circ} \mathrm{C}$. The wells were washed twice to remove any free drug and 1.5 $\mathrm{mL}$ of drug-free culture medium was added to the well. The supernatant covering the FG layer was harvested from the wells after $2,24,48$ or 72 hours by 2 different protocols. In the cumulative method (Figure 2a) four FG-drug wells were set up and the supernatants were collected for each time period from a separate well respectively. For the sequential method only (Figure 2b) one FG-drug well was made. The supernatant was collected after 2 hours of incubation and replaced by 1.5 $\mathrm{ml}$ of fresh medium and this process was repeated every 24 hours for a total of 72 hours.

\section{Direct measurement of FG drug release}

Fluorescence emission spectroscopy was performed to determine the relative concentrations of BLM and DOX in the supernatant. Measurements were carried out with a Cary Eclipse Fluorimeter, using a $1 \mathrm{~cm}$ quartz cuvette. For BLM, the excitation wavelength was set to $288 \mathrm{~nm}$, which corresponds to the maximum absorption of the compound. Fluorescence emission was recorded between $300 \mathrm{~nm}$ and $500 \mathrm{~nm}$, with the BLM emission maximum found at $345 \mathrm{~nm}$. For DOX, the excitation wavelength was tuned to the absorption maximum at $484 \mathrm{~nm}$, and fluorescence was recorded between $500 \mathrm{~nm}$ and $700 \mathrm{~nm}$. DOX exhibits a characteristic fluorescence spectrum with a maximum near $600 \mathrm{~nm}$. Relative concentrations of the drugs were determined for supernatant samples collected at different times by comparing the integrated fluorescence intensity near the emission maximum within a $10 \mathrm{~nm}$ bin.

\section{Spheroid formation}

A modified somewhat simplified method of that previously used [12] for spheroid formation was used in all experiments. F98 cells in suspension were incubated with AlPcS $_{2 a}$, at $1 \mu \mathrm{g} /$ $\mathrm{mL}$ for 2 hours, spun down and washed twice in medium to remove excess photosensitizer. The cells were used to form spheroids by a modification of the centrifugation method as previously described. Briefly, $2.5 \times 10^{3}$ AlPcS $_{2 a}$ cells in $100 \mu \mathrm{L}$ of culture medium per well were aliquoted into the wells of ultra-low attachment surface 96 -well round-bottomed plates (Corning In.,NY). The plates were centrifuged at $1000 \mathrm{~g}$ for 30 minutes. Immediately following centrifugation, the tumor cells formed into a disk shape. The plates were maintained at $37^{\circ} \mathrm{C}$ in a $5 \% \mathrm{CO}_{2}$ incubator for 24 hours to allow them to take on the usual 3-dimensionalspheroid form. The spheroids formed were uniform in size and were approximately $0.2 \mathrm{~mm}$ in diameter.

\section{$\mathrm{PCI}$}

The basic FG-PCl experimental protocol is shown in Figure 2c. The formed spheroids were incubated together with 0.1 $\mathrm{m}$ of supernatant containing FG released drug collected at increasing times. For comparison purposes, spheroids were also cultured with BLM or DOX as pure drug at increasing concentrations ranging from 0-1.2 $\mu \mathrm{g} / \mathrm{mL}$ for BLM and 0-0.1 $\mu \mathrm{g} /$ $\mathrm{mL}$ for DOX.

One hour after BLM, DOX or supernatant was added, light 

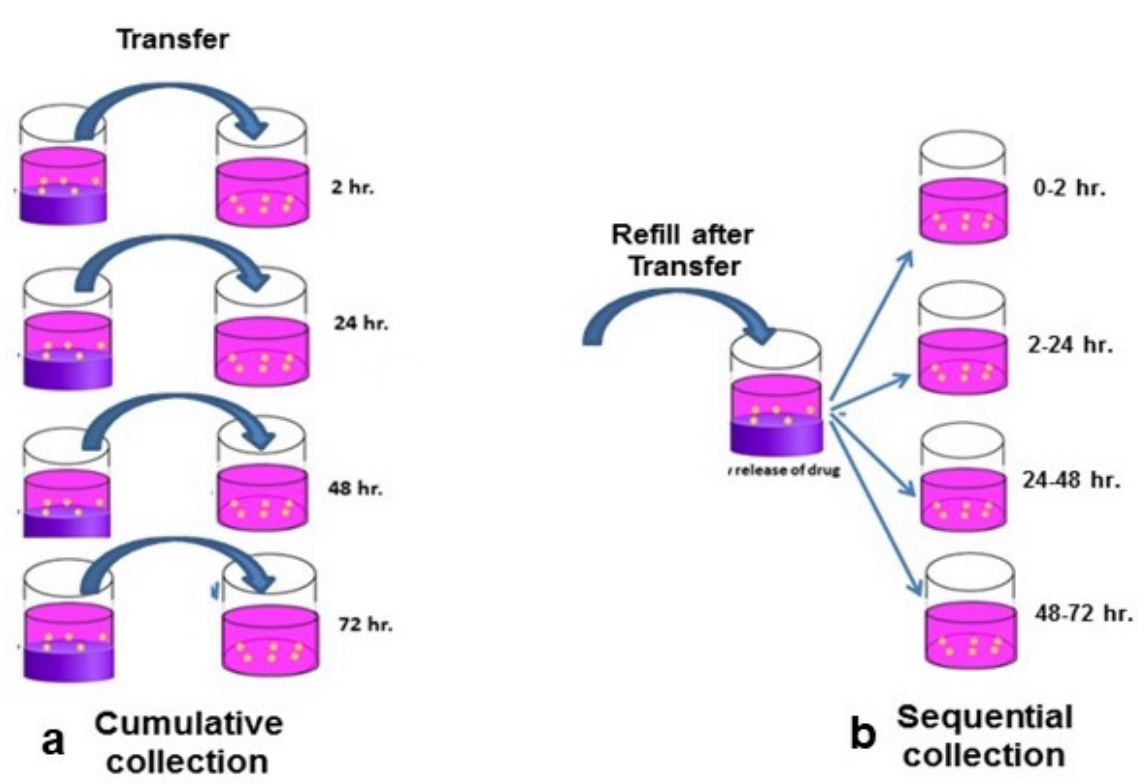

b Sequential collection

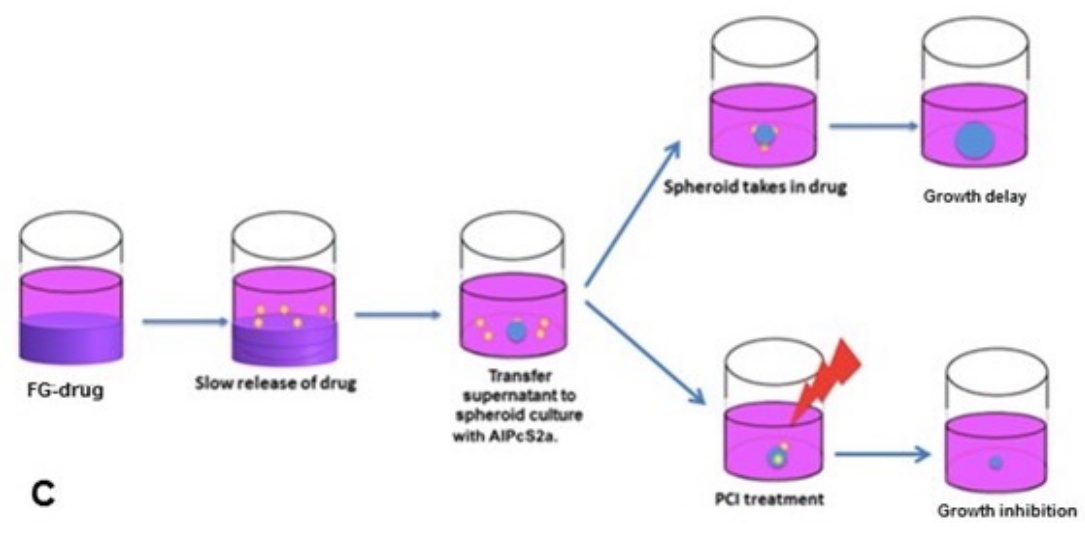

Figure 2: Overview of BLM released from FG and combined with $\mathrm{F} 98$ tumor spheroids with $\mathrm{AlPCS}_{2 \mathrm{a}}$ in two separate collection methods. a) The cumulative collection method involves the removal FG-BLM supernatant from four separate wells, each designated for the removal after 2, 24, 48 and 72 hours; b) The sequential collection method involves one well that undergoes the removal of supernatant after 2 hours. The well is then refilled with medium and removed after 24 hours. This process is repeated until 72 hours; c) FG-BLM slowly releases BLM into the supernatant, which is then combined with $\mathrm{F98}$ spheroid culture with $\mathrm{AlPCS}_{2 \mathrm{a}}$. Without $\mathrm{PCl}$, the tumor has a delayed growth, whereas $\mathrm{PCl}$ treatment shows inhibited growth.

treatment, $\mu=670 \mathrm{~nm}$, from a diode laser (Intense; New Jersey USA) at an irradiance of $2.0 \mathrm{~mW} / \mathrm{cm}^{2}$ was administered for 8.0 or $10 \mathrm{~min}$. corresponding to 0.96 or $1.2 \mathrm{~J} / \mathrm{cm}^{2}$ respectively.

Control cultures received either light treatment but no drug or FG supernatant (PDT control) or they received drug/ FG supernatant but no illumination (drug-only control).

Following PCI or PDT, the plates were returned to the incubator. Typically, 8-16 spheroids were followed for each category, in three independent experiments, for up to 14 days of incubation. Culture medium in the wells was exchanged every third day. Determination of spheroid growth was carried out by averaging two measured perpendicular diameters of each spheroid using a microscope with a calibrated eyepiece micrometer and their volume calculated assuming a perfect sphere.

\section{Statistical analysis}

All data were analyzed and graphed using Microsoft Excel. The arithmetic mean and standard error were used throughout to calculate averages and errors. Statistical significance was calculated using the Student's t-test as well as the Welch's t-test. Two values were considered distinct when their p-values were below 0.05. Synergism was calculated when analyzing $\mathrm{PCl}$ treatments compared with drug or PDT alone. The equation $\alpha=\frac{S F^{P D T} \times S F^{d r u g}}{S F^{P C I}}$ was used to determine if the $\mathrm{PCl}$ effect was synergistic, antagonistic, or additive. $\alpha$ is defined as the ratio of the cumulative effect of 2 therapies, PDT and drug, administered independently to the net effect of combining the 2 therapies at a given dose. In this scheme, SF represents the survival fraction for a specific treatment. 

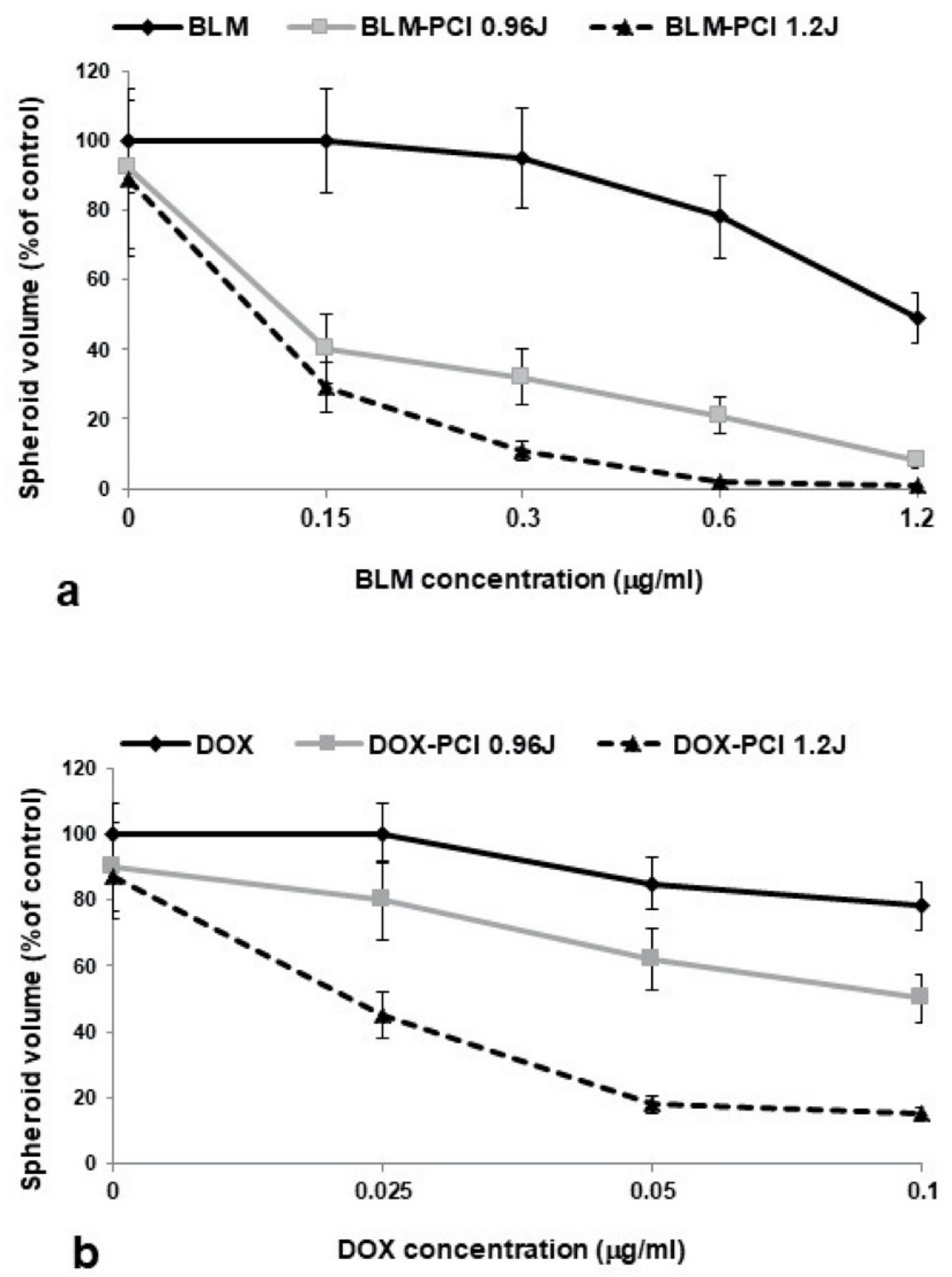

Figure 3: Growth inhibition of $\mathrm{F} 98$ glioma spheroids by free BLM or DOX with $\mathrm{PCl}$. (a) Effects of BLM-PCl at a radiance of $0,0.96$ and $1.2 \mathrm{~J} / \mathrm{cm}^{2}$ treated with BLM (0-1.2 $\left.\mu \mathrm{g} / \mathrm{mL}\right)$; (b) Effects of DOX-PCl at 0, 0.96 and $1.2 \mathrm{~J} /$ $\mathrm{cm}^{2}$. DOX concentrations varying from $0-0.1 \mu \mathrm{g} / \mathrm{mL}$.

Error bars represent standard deviation and ${ }^{*}$ represents significant differences $(p<0.05)$ when compared to controls.

If 2 treatments are to be compared, the survival fractions of each separate treatment are multiplied together and then divided by the survival fraction when both treatments were applied together. The interaction is calculated based on the dose of each treatment. The resulting number $(\alpha)$ describes the summative effect as previously described [18]. If $\alpha>1$, the result is synergistic (supra-additive). If $\alpha<1$, the result is antagonistic, and if $\alpha=1$ the result is simply additive.

\section{Results}

\section{$\mathrm{PCl}$ of BLM and DOX}

In order to compare the effects on spheroid growth of
BLM and DOX released from the FG layer experiments were performed, with and without $\mathrm{PCl}$, using increasing concentrations of free BLM or DOX. The results are shown in Figure $3 a$ and Figure $3 b$ for increasing drug concentration and light dose for both drugs. Free drug-only experiments indicated that at concentrations ranging from 0 to $1.2 \mu \mathrm{g} / \mathrm{mL}$ for BLM and 0 to $0.1 \mu \mathrm{g} / \mathrm{mL}$ for DOX in the absence of light exposure, significant growth inhibition was seen only at the highest drug concentrations tested. On the other hand, the growth inhibitory effect of both drugs was significantly enhanced by $\mathrm{PCl}$ and was clearly synergistic for both BLM and DOX at the irradiance levels examined. The results of these experiments therefore act as standard calibration data that can be used to 


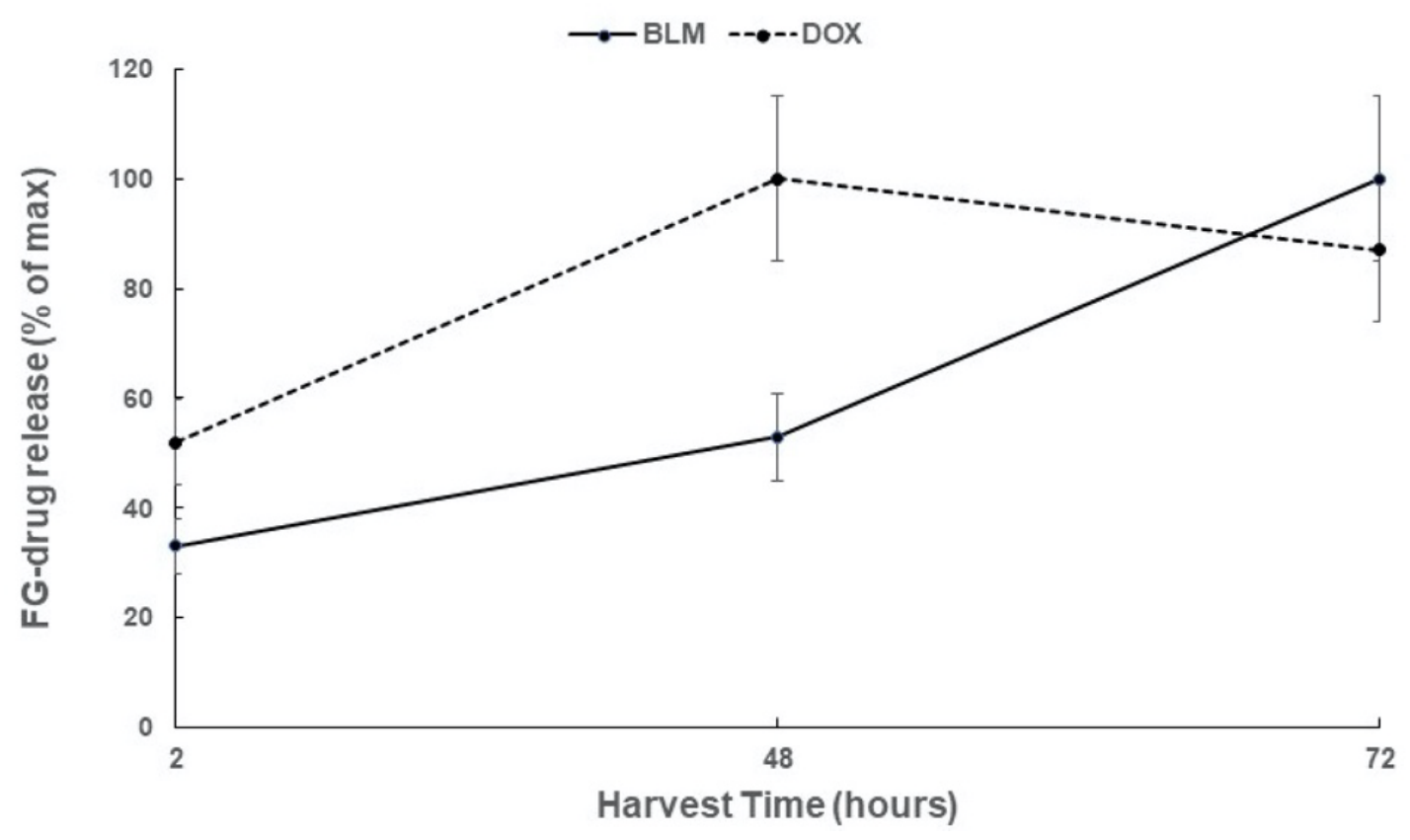

Figure 4: Direct measurement of FG BLM or DOX release by fluorescence emission spectroscopy. Time course ofcumulative release from FG- BLM or FG- DOX, after 2, 48, 72 hours and assayed by drug flourescence. FG loaded with $5 \mu \mathrm{g} / \mathrm{mL}$ of BLM or $1 \mu \mathrm{g} / \mathrm{mL}$ of DOX. The results are shown as a $\%$ of the maximum release. Error bars represent standard deviation.

determin the concentration of FG released drug.

\section{Direct determination of BLM and DOX release}

The time course of the cumulative release from FG of BLM and DOX, as assayed by drug flourescence, is shown in Figure 4. The FG was loaded with $5 \mu \mathrm{g} / \mathrm{mL}$ of BLM or $1 \mu \mathrm{g} / \mathrm{mL}$ of DOX and the results are shown as a \% of the maximum release. BLM release was more protracted ( 72 hours) compared to the release of DOX which reached a maximum after 48 hours.

\section{Effects on spheroid growth of FG-BLM/DOX PCI}

\section{Cumulative supernatant harvest}

Similar spheroid experiments as those described above for $\mathrm{PCl}$ for free BLM and DOX were performed employing supernatants containing released drug from loaded FG. The supernatants were harvested after 2, 24, 48 and 72 hours of incubation from 4 independent wells (Figure 2a). The results shown in Figure $5 a$, Figure $5 b$ and Figure $5 c$ represent spheroid growth following a 14 day period in culture. In the experiments shown in Figure $5 \mathrm{a}, 5 \mathrm{\mu g} / \mathrm{mL}$ of BLM were incorporated into the $\mathrm{FG}$. $\mathrm{PCl}$ was done at a radiance of $1.2 \mathrm{~J} / \mathrm{cm}^{2}$. As seen in Figure $5 \mathrm{a}$, in the absence of light treatment, the FG-BLM containing supernantants were not significantly inhibitory for spheroid growth for any of the harvest times examined. In contrast, spheroid growth inhibition was significantly enhanced by $\mathrm{PCl}$. Significant growth inhibition was observed even by the supernatant harvested during the first 24 hours. Supernatants harvested after 48 and 72 hours showed only a slight non significant additional increase in PCl-mediated inhibitory effects.
Figure 5b shows light micrographs of F98 spheroids from a single experiment 14 days post-treatment. Although the spheroid exposed to $\mathrm{FG}$ released $\mathrm{BLM}$ in the absence of $\mathrm{PCl}$ as shown is slightly smaller than controls, this is a growth delay effect. Examining these spheroids two days later determined that they were of equal size compared to controls (data not shown). In contrast, the significant growth inhibition by FGBLM-PCl using FG supernatants harvested after 24 hours is clearly demonstrated. The $\mathrm{PCl}$ treated spheroids are approximately the same size on day 14 as they were on day 2, indicating complete growth suppression.

The inhibitory effects of DOX, released from FG loaded with $1 \mu \mathrm{g} / \mathrm{mL}$ of the drug, weresignificantly inhibitory compared to non-treated controls for the harvest period of 24 hours (Figure $5 \mathrm{c}$ ). For the $48-72$ hour period the direct inhibitory effects decreased. This may have been due to a deterioration of the drug in the prolonged incubation in the presence of the FG layer. $\mathrm{PCl}$ of the released FG-DOX, as was the case for FG-BLM, significantly increased the inhibitory effects on spheroid growth at all of the harvest times. A slight decrease in FG-DOX PCl-mediated spheroid inhibition at the 72 hour harvest time paralled that seen for FG-DOX in the absence of $\mathrm{PCl}$.

For both BLM and DOX the effects of $\mathrm{PCl}$ were clearly synergistic at all of the harvesting intervals. $\alpha$ values for both $\mathrm{PCl}$ of BLM and DOX were calculated from the data shown over the colums in Figure $5 \mathrm{a}$ and Figure $5 \mathrm{c}$, Table 1. PCl of released drug harvested at 2, 24, 48 and 72 hours all resulted in $\alpha$ values greater than 1 , indicating a significant synergistic response $(p<0.05)$. 

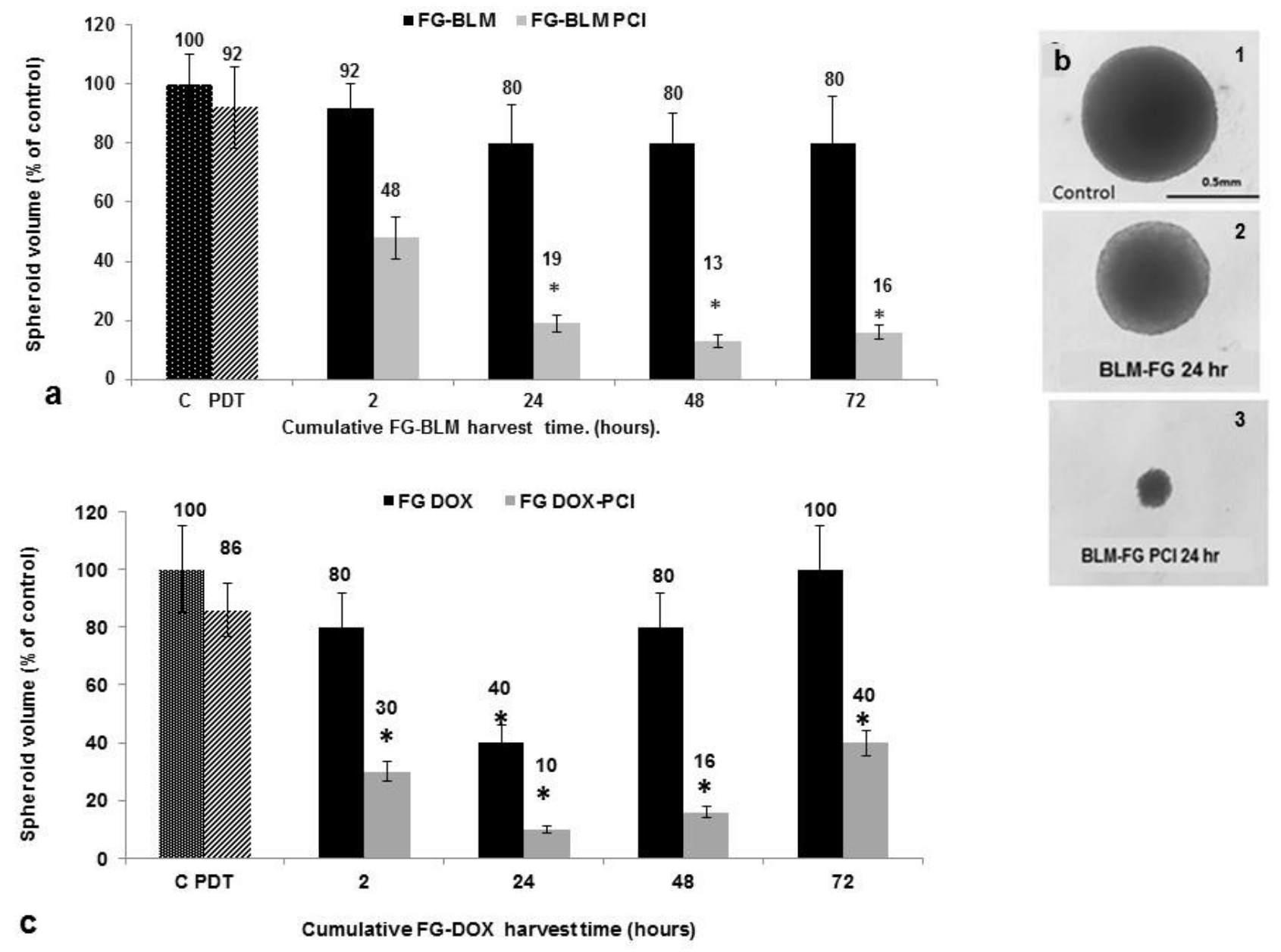

Figure 5: Effects on spheroid growth of FG-BLM or DOX with and without PCl; Cumulative supernatant harvest. FG released drug containing supernatants harvested after 2, 24, 48, 72 hours from 4 separate FG cultures. (a) Effects of FG-BLM and FG-BLM PCl on the growth of F98 spheroids; (b) Light micrograph image of F98 spheroid exposed to FG-BLM-PCI supernatants harvested after 24 hours. Spheroid growth after 14 days. 1) non-treated controls. 2) FG-BLM, 3) FG-BLM-PCI. Scale bar as shown; (c) Effects of FG-DOX and FG-DOX-PCl. Each data point value is shown over the respective column and represents mean volume of 8 spheroids in one typical experiment in a series of 3.

Error bars represent standard deviation and *represents significant differences $(p<0.05)$ when compared to controls.

Table 1: Calculated $\alpha$ for PCl of FG-BLM and FG-DOX.

\begin{tabular}{|l|l|l|l|l|}
\hline & \multicolumn{4}{|c|}{ Time (hours) } \\
\hline Treatment groups & $\mathbf{2}$ & $\mathbf{2 4}$ & $\mathbf{4 8}$ & $\mathbf{7 2}$ \\
\hline FG-BLM & $1.8^{*}$ & 3.8 & 5.6 & 4.6 \\
\hline FG-DOX & 2.3 & 3.4 & 4.3 & 2.2 \\
\hline
\end{tabular}

${ }^{*} \alpha$ value. $\alpha$ is defined as the ratio of the cumulative effect of 2 therapies, PDT and drug, administered independently to the net effect of combining the 2 therapies at a given dose. If $\alpha>1$, the result is synergistic (supra-additive). If $\alpha<1$, the result is antagonistic, and if $\alpha=1$ the result is simply additive.
In the sequential harvest method, the overlying medium is harvested after $2,24,48$, and 72 hours and exchanged for fresh medium after 2, 24 and 48 hours as depicted in Figure $2 \mathrm{~b}$. The results shown in Figure $6 \mathrm{a}$ and Figure $6 \mathrm{~b}$ represent spheroid growth following a 14 day period in culture. In the experiments shown in Figure $6 \mathrm{a}, 5 \mathrm{\mu g} / \mathrm{mL}$ of BLM were incorporated into the $\mathrm{FG}$. $\mathrm{PCl}$ was done at a radiance of $1.2 \mathrm{~J} / \mathrm{cm}^{2}$. As shown in the figure, in the absence of light treatment $(\mathrm{PCl})$, the FG-BLM containing supernantants were only slightly inhibitory for spheroid growth for the supernatant harvested after 2 hours. No inhibition of growh was seen for any of the harvest times examined compared to control cultures ( $P$ > 0.1 ). In contrast, spheroid growth inhibition was significantly enhanced by $\mathrm{PCl}$. Significant growth inhibition was observed by the supernatant harvested during the first 24 hours. Supernatants harvested after 48 and 72 hours showed no addi- 

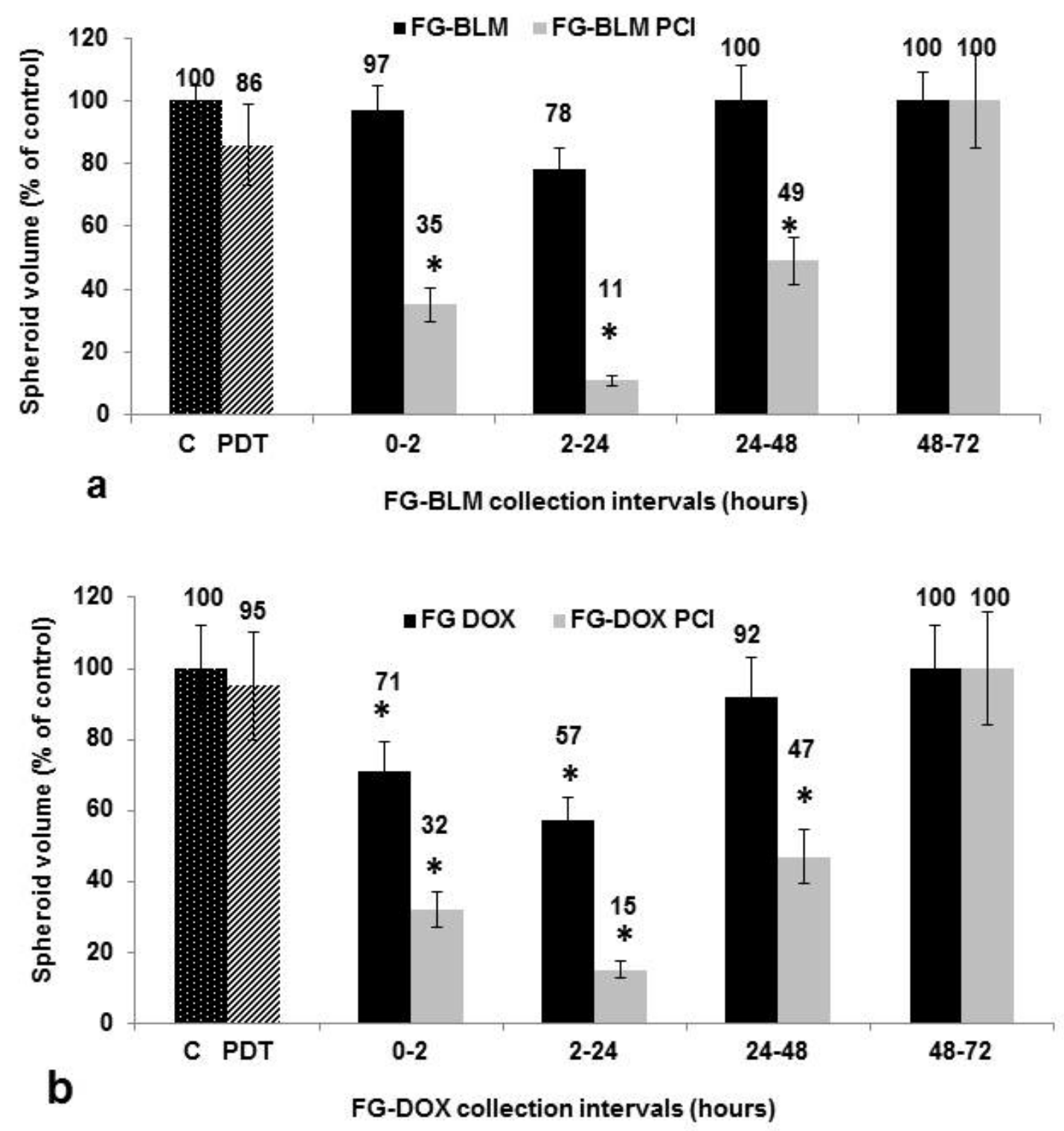

Figure 6: Effects on spheroid growth of FG-BLM or DOX with and without PCl; Sequential supernatant harvest. FG released drug containing supernatants harvested after 2, 24, 48, 72 hours from 1 FG culture with medium replacement after each harvest. (a) Effects of FG-BLM and FG-BLM PCl on the growth of F98 spheroids; (b) Effects of FG-DOX and FG-DOX PCl sequential method on the growth of F98 spheroids. Each data point value shown over the respective column and represents mean volume of 8 spheroids in one typical experiment in a series of 3 experiments.

Error bars represent standard deviation and * represents significant differences $(p<0.05)$ when compared to controls.

tional significant PCl-mediated inhibitory effects. In contrast, the inhibitory effects of DOX released from FG loaded with 1 $\mu \mathrm{g} / \mathrm{mL}$ of the drug were signficanly inhibitory at the 24 hour harvest period, compared to non-treated controls. For all of the other harvest periods (Figure $6 \mathrm{~b}$ ) little effect of DOX could be demonstrated in the absence of light treatment. $\mathrm{PCI}$ of the released FG-DOX, significantly increased the inhibitory effects on spheroid growth for the first 48 hours of harvest. No growth inhibition with or without PCl was detected for the supernatants harvested after 72 hours indicating alow drug concentration.

\section{Estimated FG-drug release}

Using the data of the effects of free drug or FG-drug- PCl on spheroid growth shown in Figure 3, Figure 5, and Figure 6 the drug concentrations released from FG for both BLM and DOX can be estimated. The results for both harvest protocols (cumulative or sequential) can be seen in Figure 7a and Figure $7 \mathrm{~b}$. For the cumulative harvest protocol, FG-BLM supernatants harvested at 2 hours gave a spheroid volume reduced to approximately $35 \%$ of control values (Figure $5 \mathrm{a}$ ). This is equivalent to that seen with a free BLM concentration of $0.15 \mu \mathrm{g} /$ 

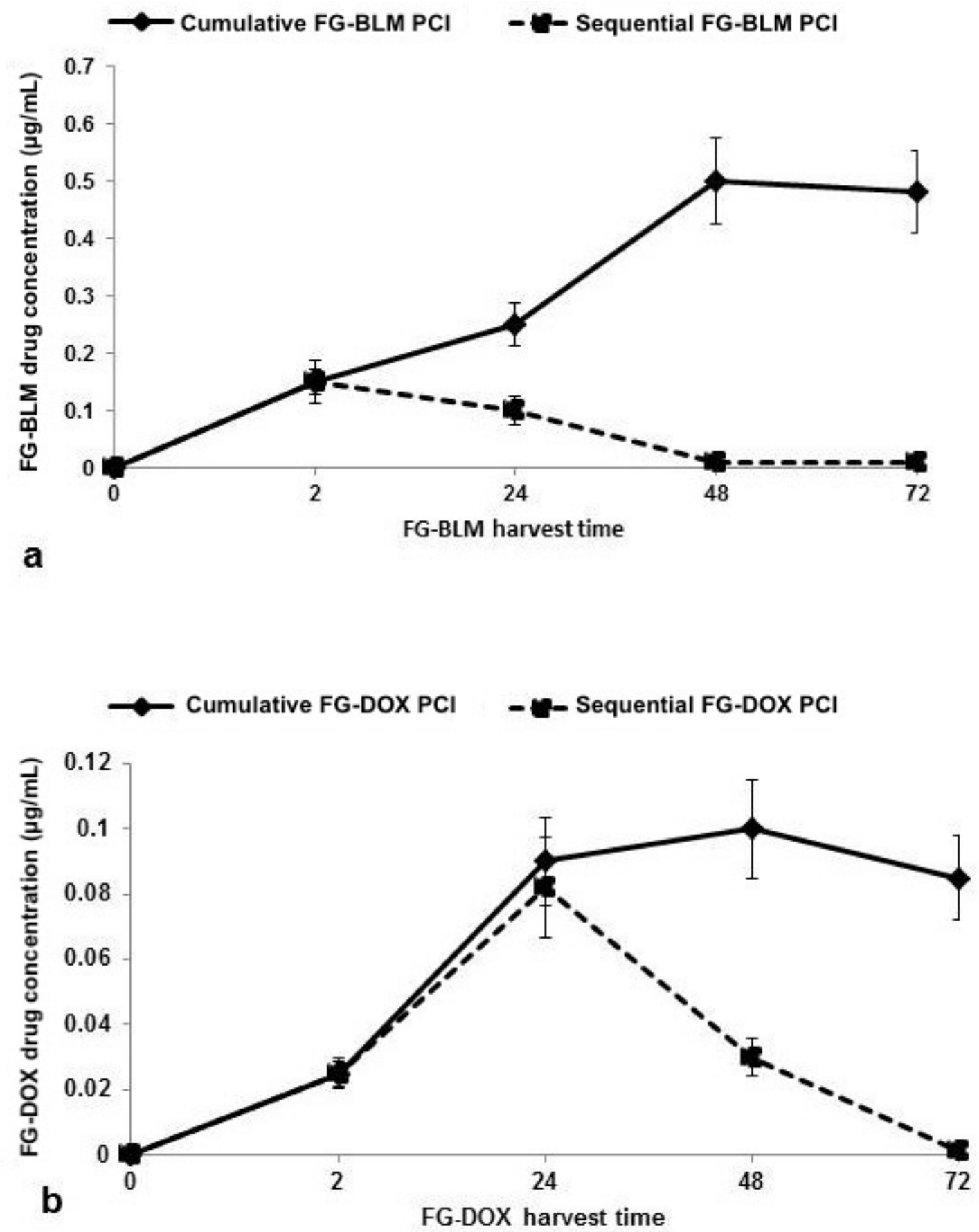

Figure 7: Estimated FG-drug release determined by effect on spheroid growth. (a) BLM concentrations calculated using free BLM effects compared to effects by FG-BLM; (b) DOX concentrations calculated using free DOX effects compared to effects by FG-DOX The results for both harvest protocols: Cummulative, solid lines or sequential, dashed lines are shown. Error bars represent standard deviation.

$\mathrm{mL}$ (Figure 3a). At FG-BLM harvested at 24, 48 and 72 hours, the concentration of BLM could be estimated at around 0.35 and $0.4-0.5 \mu \mathrm{g} / \mathrm{mL}$ respectively. Using the data from Figures $3 \mathrm{~b}$ and Figures $5 \mathrm{c}$, similar estimates for FG-DOX PCl gave a drug concentration of 0.055 and $0.095 \mu \mathrm{g} / \mathrm{mL}$ for FG-DOX supernatants harvested at 24 and 48 hours respectively with a slight decrease shown for the 72 hour harvest time. For the sequential harvesting protocol, assuming a FG-drug/supernatant equilibrium, the drug concentration in the $F G$ layer will be reduced to $20 \%$ of its initial concentration for each medium exchange. This is clearly demonstrated for the time course of the estimated concentration of both drug (dashed lines Figure 7).

\section{Discussion}

Employing the cumulative supernatant harvest system used in these experiments, it is assumed that, after a sufficient incubation interval, an equilibrium drug concentration 
Citation: Nguyen L, Shin D, Le MT, et al. (2020) Local Drug Delivery by Fibrin Glue for Glioma Treatment: Enhancing Drug Efficacy by Photochemical Internalization (PCI). Insights Neurooncol 3(1):31-42

will be reached between the FG layer and the surrounding culture medium. In the experimental protocol used here, the drug containing FG layer was $0.4 \mathrm{~mL}$ in volume while the overlaying supernatant was $1.5 \mathrm{~mL}$. It is assumed that the drug will slowly diffuse from the FG into the supernatant. At equilibrium, the final drug concentration in the supernatant would therefore represent approximately $20 \%(0.4 / 1.9 \mathrm{~mL})$ of the initial drug concentration, corresponding to $1 \mu \mathrm{g} / \mathrm{mL}$ for BLM and $0.2 \mu \mathrm{g} / \mathrm{mL}$ for DOX, respectively. The maximum FG released drug concentrations shown in Figure 4 therefore probably represent these values. In addition, $0.1 \mathrm{~mL}$ of the supernatants were added to $0.1 \mathrm{~mL}$ in the well containing the spheroid, reducing the final theoretical maximum drug concentration influencing spheroid growth to $0.5 \mu \mathrm{g} / \mathrm{mL}$ for BLM and $0.1 \mu \mathrm{g} / \mathrm{mL}$ for DOX.

The results shown in Figure 4 (direct drug concentration) and Figure 7 (estimated drug concentration) although similar in time course are not identical. This is in all probability caused by the non-linear effect PCl mediated drug induced inhibition of spheroid growth as seen in Figure 3 for free drug. Increasing the drug concentration over a threshold level does not cause a related increase in spheroid growth inhibition. The increase in BLM concentration between 48 and 72 hours as shown in Figure 4 is therefore not reflected in the results shown in Figure 7a due to this "drug saturation" effect.

Although BLM and DOX are commonly used in a number of standard cancer therapies, they have been found to have limited use for the treatment of gliomas. Due to its hydrophilic nature and relatively large size, BLM has very limited penetration through the BBB and plasma cell membrane $[19,20]$. Although this is a clear disadvantage in terms of treatment efficacy, normal brain cells are protected from their toxic effects since these drugs would not retrograde re-enter the systemic blood stream. This contrasts with lipophilic chemotherapeutic agents like BCNU or TMZ, now in use clinically. Thus, BLM or DOX could be highly effective drugs for treatment of glioblastoma multiforme (GBM) in combination with local delivery strategies that increase the drug concentrations at the tumor site, bypassing the BBB and avoiding systemic side effects.

Enhanced efficacy of both BLM and DOX by PCl has been previously demonstrated on a variety of cancer cell types including glioma cells $[12,14]$. The data shown in Figure 5 and Figure 6 clearly indicate a significant increase in PCl-mediated FG-BLM or FG-DOX toxicity compared to either PDT or drug alone at the light levels and drug concentrations used. Due to its hydrophilic character, BLM is actively taken up into cells by endocytosis. Its poor ability to escape from endosomes leads to inactivation by hydrolytic enzymes and complexing molecules in secondary endosomes. In contrast, DOX in its neutral form is membrane permeable but relatively membrane impermeable in the acidic environment of lysosomes or recycling endosomes where it will be sequestered and inactivated within these intra-cellular compartments [21,22]. However, if released, these drugs quickly diffuse into the nucleus where they have a significant toxic effect. These characteristics make $\mathrm{BLM}$ or DOX well suited for use together with $\mathrm{PCl}$, where the drug is selectively released from endosomal/lysosomal en- trapment into the cell cytosol and nucleus.

$\mathrm{PCl}$ has been proven effective employing light treatment protocols either before or after drug administration [23,24]. In the case of DOX-PCl, it appears that the light before sequence is more effective than the light after $[14,25]$. In the experiments reported here, free or FG-released DOX was added to the cultures 24 hours after spheroid formation. Following a 1-hour incubation period, the initiation of light treatment was commenced. Since additional wash cycles were not used to remove any remaining DOX from the cultures, it is assumed that active drug was still available post-light treatment and therefore this protocol can be considered a combined light "after" and light "before" sequence. The pronounced synergistic effect of PCI-DOX on F98 spheroids was likely due to a combination of both facilitating its endo-lysosomal release (light after sequence) and by preventing lysosomal uptake of DOX (light before sequence).

Several other types of hydrogels besides FG have been studied for the local delivery and slow release of chemotherapeutic drugs in the tumor resection cavity for brain tumors in experimental animals [26]. In particular, Bastiancich and co-workers have developed a hydrogel made of lipid nanocapsules loaded with lauroyl-gemcitabine $[4,27,28]$. They have evaluated its effect in a resected GBM rat model and found that after local peri-surgical administration (in the resection cavity), the formation of recurrences of $9 \mathrm{~L}$ tumors was delayed [27].

Fibrin glue though, has several characteristics that make it an attractive alternative to other forms of hydrogels. Firstly, thrombin and fibrinogen are naturally occurring substance in the coagulation process and are of low toxicity. FG has been used in surgical procedures for decades and is widely clinically approved. In addition, the parameters of the components can be modified to change the gel's structure, mechanical properties, and degradation [29]. FG is also easily molded to coat the walls of the resection cavity.

In the formulation of fibrin glue for chemotherapeutic agents used in cancer drug delivery, exogenous drugs are added to one of the components before addition of the other, allowing the drug to be distributed throughout the solution before cross linking, as was done in the experiments shown in Figure 4 and Figure 5. Although FG has been described for drug delivery for a variety of agents, it is the local release of chemotherapeutic drugs that is of interest here. Anai, et al. investigated FG as a drug delivery system for the local administration of Temozolomide (TMZ) in mice bearing subcutaneous tumors induced by the injection of malignant glioma cell lines. Slabs of gelled TMZ-containing FG were surgically placed in contact with the tumors. In mice treated with a combination of per-oral TMZ plus gelled FG-TMZ the tumors tended to be smaller than in mice treated with TMZ-FG or per-oral TMZ alone [7]. Furthermore, gelled FG-TMZ placed directly on the brain of living mice caused no significant tissue damage either in the acute or chronic phase. Although TMZ is extensively used in post-operative clinical treatment in patients, it was not evaluated in our study since it has been shown that its therapeutic effects are not significantly enhanced by $\mathrm{PCl}$ [30]. 


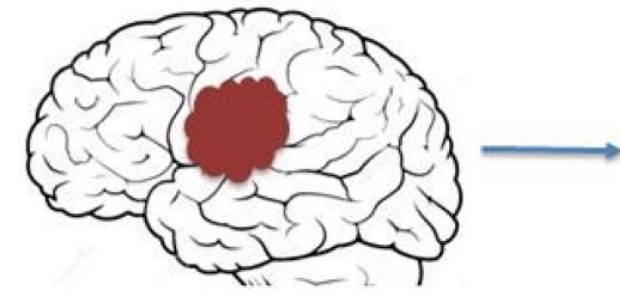

a brain with tumor
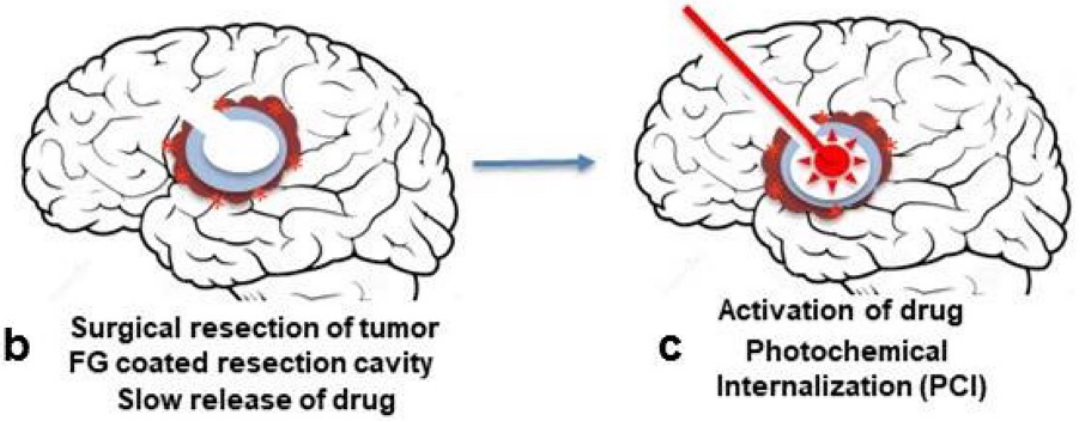

Figure 8: Schematic illustration of proposed translation of loaded FG-PCl protocol. (a) Brain with tumor; (b) drug diffusion in post-surgical resection cavity lined with loaded FG; (c) intra-cavity light application (PCI) via an indwelling applicator.

Formulations of fibrin glue can also include anti-fibrinolytic agents such as aprotinin to delay or slow fibrinolysis, which in turn would lead to slower drug release [31,32]. Yoshida, et al. demonstrated that the release of 5-FU and mitomycin were released relatively rapidly, independent of aprotinin, compared to enocitabine where drug release was slowed in the presence of aprotinin [5].

In all probability, the release profile of DOX and especially BLM seen in the experiments reported in the present study would benefit if prolonged past the 48-hour time point. Kitazawa, et al. examined the release kinetics of DOX from FG or a composite gel of fibrin with sodium alginate [33]. The in vitro mean release times of DOX from FG and FG containing sodium alginate were 8.7 hours, and 81 hours, respectively. This clearly indicated a significant sustained release of DOX in the presence of sodium alginate. All in all, these previously reported studies clearly illustrated the potential of fibrin glue as a carrier for combined localized delivery systems such as drugs, nanoparticles or composite gels.

The combination of intra-cavity FG or hydrogel slow-release drug delivery combined with $\mathrm{PCl}$ therefore has the potential of by passing the BBB in a targeted confined area thus allowing increased chemotherapeutic efficacy while reducing treatment side effects. This approach can be readily translated to in vivo animal experiments and since $\mathrm{FG}$ is widely clinically approved, eventually to patient treatment protocols (Figure 8). As shown in Figure 8, following tumor resection, the resection cavity is coated with drug/photosensitizer loaded FG. The FG slowly dissolves releasing the drug. Light treatment is done through a balloon applicator implanted during the initial cytoreductive surgery and positioned in the center of FG lined resection cavity. Indwelling light/radiation applicators have been temporarily implanted in resection cavities during surgery, remaining in place for up to one week before removal [34-36].

\section{Conclusion}

The results of the present study show that active drug was released from $F G$ layers for an extended time period. The growth inhibition caused by either FG released BLM or DOX was significantly enhanced by $\mathrm{AlPcS}_{2 a}$ mediated PCI. AlPcS ${ }_{2 \mathrm{a}^{\prime}}$ released from $\mathrm{FG}$, together with light treatment could also provide for effective PDT.

\section{Funding}

The Norwegian Radium Hospital Research Foundation. Grant nr. SE. 1305/1503.

\section{Conflict of Interest}

All of the authors declare that she/he has no conflict of interest.

\section{Ethical Approval}

This article does not contain any studies with human participants or animals performed by any of the authors.

\section{Acknowledgments}

The authors are grateful for the support from the Norwegian Radium Hospital Research Foundation.

\section{References}

1. Chamberlain MC (2011) Radiographic patterns of relapse in glioblastoma. J Neurooncol 101: 319-323.

2. Dobelbower MC, Burnett lii OL, Nordal RA, et al. (2011) Patterns of failure for glioblastoma multiforme following concurrent radiation and temozolomide. J Med Imaging Radiat Oncol 55: 77-81.

3. Lawson HC, Sampath P, Bohan E, et al. (2007) Interstitial chemotherapy for malignant gliomas: The Johns Hopkins experience. J Neurooncol 83: 61-70.

4. Bastiancich C, Danhier P, Préat V, et al. (2016) Anticancer drug-loaded hydrogels as drug delivery systems for the local treatment of glioblastoma. J Control Release 243: 29-42.

5. Yoshida H, Yamaoka Y, Shinoyama M, et al. (2000) Novel drug delivery system using autologous fibrin glue--release properties of anti-cancer drugs. Biol Pharm Bull 23: 371-374.

6. Spicer PP, Mikos AG (2010) Fibrin glue as a drug delivery system. J Control Release 148: 49-55.

7. Anai S, Hide T, Takezaki T, et al. (2014) Antitumor effect of fibrin glue containing temozolomide against malignant glioma. Cancer Sci 105: 83-91.

8. Berg K, Selbo PK, Prasmickaite L, et al. (1999) Photochemical in- 
Citation: Nguyen L, Shin D, Le MT, et al. (2020) Local Drug Delivery by Fibrin Glue for Glioma Treatment: Enhancing Drug Efficacy by Photochemical Internalization (PCI). Insights Neurooncol 3(1):31-42

ternalization: A novel technology for delivery of macromolecules into cytosol. Cancer Res 59: 1180-1183.

9. Weyergang A, Berstad ME, Bull-Hansen B, et al. (2015) Photochemical activation of drugs for the treatment of therapy-resistant cancers. Photochem Photobiol Sci 14: 1465-1475.

10. Sosic L, Selbo PK, Kotkowska ZK, et al. (2020) Photochemical internalization: Light paves way for new cancer chemotherapies and vaccines. Cancers (Basel) 9: 165.

11. Berg K, Dietze A, Kaalhus O, et al. (2005) Site-specific drug delivery by photochemical internalization enhances the antitumor effect of bleomycin. Clin Cancer Res 11: 8476-8485.

12. Mathews MS, Blickenstaff JW, Shih EC, et al. (2012) Photochemical internalization of bleomycin for glioma treatment. Biomed Opt 17.

13. Lou PJ, Lai PS, Shieh MJ, et al. (2006) Reversal of doxorubicin resistance in breast cancer cells by photochemical internalization. Int J Cancer 119: 2692-2698.

14. Shin D, Christie C, Ju D, et al. (2018) Photochemical internalization enhanced macrophage delivered chemotherapy. Photodiagnosis Photodyn Ther 21: 156-162.

15. Sultan AA, Jerjes W, Berg K, et al. (2016) Disulfonated tetraphenyl chlorin (TPCS2a)-induced photochemical internalisation of bleomycin in patients with solid malignancies: A phase 1, dose-escalation, first-in-man trial. Lancet Oncol 17: 1217-1229.

16. Norum OJ, Giercksky KE, Berg K (2009) Photochemical internalization as an adjunct to marginal surgery in a human sarcoma model. Photochem Photobiol Sci 8: 758-762.

17. Madsen SJ, Sun CH, Tromberg BJ, et al. (2006) Multicell tumor spheroids in photodynamic therapy. Lasers Surg Med 38: 555564.

18. Drewinko B, Loo TL, Brown B, et al. (1976) Combination chemotherapy in vitro with adriamycin. Cancer Biochem Biophys 1: 187-195.

19. Linnert M, Gehl J (2009) Bleomycin treatment of brain tumors: An evaluation. Anticancer Drugs 20: 157-164.

20. Salford LG, Persson BR, Brun A, et al. (1993) A new brain tumor therapy combining bleomycin with in vivo electropermeabilization. Biochem Biophys Res Commun 194: 938-943.

21. Mayer LD, Bally MB, Cullis PR (1986) Uptake of adriamycin into large unilamellar vesicles in response to a $\mathrm{pH}$ gradient. Biochim Biophys Acta 857: 123-126.

22. Coley HM, Amos WB, Twentyman PR, et al. (1993) Examination by laser scanning confocal fluorescence imaging microscopy of the subcellular localisation of anthra-cyclines in parent and multidrug resistant cell lines. Br J Cancer 67: 1316-1323.
23. Prasmickaite L, Hogset A, Selbo PK, et al. (2002) Photochemical disruption of endocytic vesicles before delivery of drugs: A new strategy for cancer therapy. Br J Cancer 86: 652-657.

24. Berstad MB, Weyergang A, and Berg K (2012) Photochemical internalization (PCI) of HER2-targeted toxins: Synergy is dependent on the treatment sequence. Biochim Biophys Acta 1820: 1849-1858.

25. Lai PS, Lou PJ, Peng CL, et al. (2007) Doxorubicin delivery by polyamidoamine dendrimer conjugation and photochemical internalization for cancer therapy. J Control Release 122: 39-46.

26. Madsen SJ, Angell-Petersen E, Spetalen S, et al. (2006) Photodynamic therapy of newly implanted glioma cells in the rat brain. Lasers Surg Med 38: 540-548.

27. Bastiancich C, Lemaire L, Bianco J, et al. (2018) Evaluation of lauroyl-gemcitabine-loaded hydrogel efficacy in glioblastoma rat models. Nanomedicine 13: 1999-2013.

28. Bastiancich C, Bozzato E, Luyten U, et al. (2019) Drug combination using an injectable nanomedicine hydrogel for glioblastoma treatment. Int J Pharm 559: 220-227.

29. Sierra DH (1993) Fibrin sealant adhesive systems: A review of their chemistry, material properties and clinical applications. J Biomater Appl 7: 309-352.

30. Gederaas OA, Hauge A, Ellingsen PG, et al. (2015) Photochemical internalization of bleomycin and temozolomide - in vitro studies on the glioma cell line F98. Photochem Photobiol Sci 14: 13571366.

31. Pipan CM, Glasheen WP, Matthew TL, et al. (1992) Effects of antifibrinolytic agents on the life span of fibrin sealant. J Surg Res 53: 402-407.

32. Beduschi R, Beduschi MC, Wojno KJ, et al. (1999) Antifibrinolytic additives to fibrin glue for laparoscopic wound closure in urinary tract. J Endourol 13: 283-287.

33. Kitazawa H, Sato H, Adachi I, et al. (1997) Microdialysis assessment of fibrin glue containing sodium alginate for local delivery of doxorubicin in tumor-bearing rats. Biol Pharm Bull 20: 278281.

34. Johannesen TB, Watne K, Lote K, et al. (1999) Intracavity fractionated balloon brachytherapy in glioblastoma. Acta Neurchiurica 141: 127-133.

35. Madsen SJ, Sun CH, Tromberg B, et al. (2001) Development of a novel balloon applicator for optimizing light delivery in photodynamic therapy. Lasers in Surgery and Medicine 29: 406-412.

36. Eljamel MS, Goodman C, Moseley H (2008) ALA and photofrin fluorescence-guided resection and repetitive PDT in glioblastoma multiforme: A single center phase III randomized controlled trial. Lasers Med Sci 23: 361-367.

DOI: $10.36959 / 828 / 334$

Copyright: (C) 2020 Nguyen L, et al. This is an open-access article distributed under the terms of the Creative Commons Attribution License, which permits unrestricted use, distribution, and reproduction in any medium, provided the original author and source are credited. 